\title{
External losses in high-bypass turbo fan air engines
}

\section{Henning Struchtrup*}

ETH Zürich, Department of Materials, Polymer Physics, HCI H 539,

CH-8093 Zürich, Switzerland

Department of Mechanical Engineering, University of Victoria, P.O. Box STN CSC 3055,

Victoria BC V8W 3P6, Canada

E-mail: struchtr@uvic.ca

${ }^{*}$ Corresponding author

\section{Gwynn J. Elfring}

Department of Mechanical Engineering, University of Victoria, P.O. Box STN CSC 3055,

Victoria BC V8W 3P6, Canada

E-mail: gelfring@uvic.ca

\begin{abstract}
The external irreversible losses of air engines, due to equilibration of the hot and fast exhaust with the environment, are discussed based on the second law of thermodynamics. The effect of the bypass ratio on thermomechanical exergy destruction in the exhaust stream is demonstrated. The analysis gives a strong motivation for the use of high bypass turbo fan engines in modern aircraft.
\end{abstract}

Keywords: bypass air engine; second law analysis; engineering education.

Reference to this paper should be made as follows: Struchtrup, H. and Elfring, G.J. (xxxx) 'External losses in high: bypass turbo fan air engines' Int. J. Exergy, Vol. x, No. x, pp.xxx-xxx.

Biographical notes: Henning Struchtrup is an Associate Professor in the Department of Mechanical Engineering at the University of Victoria, Canada, where he teaches thermodynamics. His main research interests are in non-equilibrium thermodynamics and kinetic theory of gases, with applications to microscale flows.

Gwynn J. Elfring received his MASc in Mechanical Engineering at University of Victoria in 2007. His research interests lie in theoretical and applied thermodynamics, with applications to fuel cells and sustainable energy conversion. 


\section{Introduction}

In undergraduate courses on thermodynamics and energy conversion we teach our students that process performance is improved when the irreversible losses in the process, i.e., the amount of entropy generation, are reduced (Cengel and Boles, 2002; Moran and Shapiro, 1999; Sonntag et al., 2003). All energy conversion devices suffer from internal and external losses. Internal losses include for instance compressor and turbine irreversibilities (as expressed through isentropic efficiencies), and are routinely covered in typical undergraduate courses. External irreversibilities are only covered occasionally, although they might give excellent insight into system performance.

A particular case of external irreversibility is the equilibration of the hot and fast exhaust of air engines with the environment (which is cold, and at rest). In this note it is shown that reduction of external irreversibilities is achieved in bypass turbofan engines. This provides an excellent argument - based in thermodynamics! - for building bypass engines. The usual argument uses the Froude efficiency (Cumpsty, 2003) and thus is purely mechanical. The bestselling thermodynamics textbooks on the North-American market, including Cengel and Boles (2002), Moran and Shapiro (1999) and Sonntag et al. (2003), do not provide a discussion based on physical laws, but only state in words that bypass engines are more efficient. Previous studies in the literature emphasise the exergy destruction within components of a turbofan engine (Turgut et al., 2007) or alternatively the exergy requirements for lift (Paulus and Gaggioli, 2003) or environmental control of the aircraft (Ordonez and Bejan, 2003). Here it is shown how the bypass ratio can be used to minimise the thermomechanical exergy destroyed in the exhaust stream of a bypass turbofan engine.

The second-law based argument presented below makes the topic accessible for thermodynamic courses on the undergraduate level. The treatment is quite instructive, since it considers the air engine in two different frames of reference, namely the rest frame of the engine, and the rest frame of the air that feeds the engine. We also provide a rather general calculation (with no detailed values) of how the processes in a (reversible) bypass engine can be computed. Most textbooks do not provide problems on bypass engines, and this section should make it easy for instructors to design their own problems.

\section{Basic equations}

Our discussion requires only basic undergraduate thermodynamics for steady state open systems with one inlet and one exit. We state the basic equations, to fix the notation which should agree with most textbooks.

The first law for a one-inlet ( $i$ )/one-exit $(e)$ steady state open system reads

$$
\dot{m}\left(h_{e}-h_{i}+\frac{1}{2}\left(\mathrm{v}_{e}^{2}-\mathrm{v}_{i}^{2}\right)\right)=\dot{Q}-\dot{W}
$$

where $h$ denotes (specific) enthalpy, $\mathbf{v}$ denotes (average) flow velocity, $\dot{m}$ is the mass flow through the device, $\dot{Q}$ and $\dot{W}$ are the rate of heat exchange and the power, respectively; external fields (e.g., gravity) are not considered. 
The second law for one-inlet/one-exit systems in steady state reads

$$
\dot{m}\left(s_{e}-s_{i}\right)-\sum_{k} \frac{\dot{Q}_{k}}{T_{k}}=\dot{S}_{\mathrm{gen}} \geq 0
$$

where $S$ is specific entropy, $\dot{Q}_{k}$, with $\sum_{k} \dot{Q}_{k}=\dot{Q}$, is the heat transfer rate for heat transferred at a location where the boundary temperature is $T_{k}$, and $\dot{S}_{\text {gen }}$ is the - non-negative - entropy production rate.

For a flow that exchanges heat only with the environment at $T_{0}$, we have $\sum_{k} \dot{Q}_{k}=\dot{Q}_{0}$ and elimination of $\dot{Q}_{0}$ between the first and second law yields the work per unit mass for such a system as

$$
w=\frac{\dot{W}}{\dot{m}}=\left(h_{i}-h_{e}-T_{0}\left(s_{i}-s_{e}\right)+\frac{1}{2}\left(\mathbf{v}_{i}^{2}-\mathbf{v}_{e}^{2}\right)\right)-T_{0} \frac{\dot{S}_{\mathrm{gen}}}{\dot{m}} .
$$

Specific flow exergy $\psi$ of a flow is defined as the amount of work per unit mass that could be extracted by bringing a flow at $T, p, \mathrm{~V}$ to equilibrium with the environment in a reversible process. In this case $h_{i}=h, s_{i}=s$ and $\mathrm{v}_{i}=\mathrm{V}$ refer to the actual state of the flow, while $h_{e}=h_{0}, s_{e}=s_{0}$ and $\mathbf{v}_{i}=\mathbf{v}_{0}$ refer to the values in equilibrium with the environment, which is at pressure $p_{0}$ and temperature $T_{0}$, and moves with velocity $\mathbf{V}_{0}$. For an observer resting in the environment we have $\mathrm{v}_{0}=0$. Since $\dot{S}_{\text {gen }}=0$ in a reversible process, we identify flow exergy from equation (3) as

$$
\psi=h-h_{0}-T_{0}\left(s-s_{0}\right)+\frac{1}{2}\left(\mathbf{v}^{2}-\mathbf{v}_{0}^{2}\right) .
$$

If a flow is expelled into the environment and no work is harvested, as it happens with the exhaust of an air engine, the exergy $\psi$ leaves the engine and is destroyed through dissipation into the environment. Thus, the amount of work lost to dissipation is directly related to the exergy output; from equation (3) with $w=0$ we identify

$$
T_{0} \dot{S}_{\text {gen }}=\dot{m} \psi
$$

\section{Aircraft engines}

Aircraft engines accelerate the flow to produce thrust, which is the force to push the airplane. We consider an airplane moving with the velocity $\mathbf{v}_{A}$ through ambient air, and an air engine accelerating the flow to $\mathbf{v}_{E}$ measured with respect to the engine. Then the thrust is given by the change of momentum of the air that passes the turbine,

$$
F=\dot{m}\left(\mathbf{v}_{E}-\mathbf{v}_{A}\right) .
$$

The propulsive power of the engine is given by the product of force and airplane velocity,

$$
\dot{W}_{P}=F \mathbf{v}_{A}=\dot{m}\left(\mathbf{v}_{E}-\mathbf{v}_{A}\right) \mathbf{v}_{A} .
$$

A standard air turbine that draws air from the environment (state 0 ) operates on the series of processes detailed in Table 1 and Figure 1. 
Table 1 Standard air turbine processes

\begin{tabular}{lll}
\hline $0-1$ & Adiabatic diffuser & $h_{1}=h_{0}+\frac{1}{2} \mathbf{v}_{A}^{2}$ \\
$1-2$ & Adiabatic compressor & $w_{C}=h_{1}-h_{2}$ \\
$2-3$ & Isobaric heating (combustion) & $q_{\text {in }}=h_{3}-h_{2}$ \\
$3-4$ & Adiabatic turbine & $w_{T}=-w_{C}=h_{3}-h_{4}$ \\
$4-5$ & Adiabatic nozzle & $\mathbf{v}_{E}=\sqrt{2\left(h_{4}-h_{5}\right)}$ \\
\hline
\end{tabular}

Figure 1 Standard air turbine jet engine

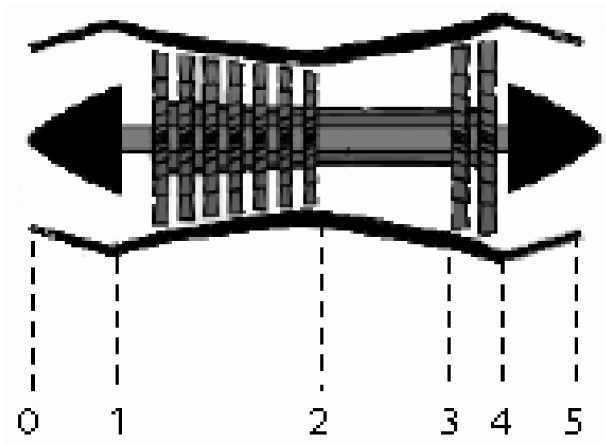

The diffuser decelerates the flow to increase the pressure, thus lowering the work $w_{C}$ required for compression. The compressed air is heated to $T_{3}$ by providing the heat $q_{\text {in }}$ from combustion of a fuel. The turbine is used solely to drive the compressor $\left(w_{T}=-w_{c}\right)$, and the hot combustion product is expanded and accelerated in a Laval nozzle to the exit state 5 .

The first law equation (1) for the whole engine (0-5) reduces to

$$
h_{5}-h_{0}+\frac{1}{2} \mathbf{V}_{E}^{2}-\frac{1}{2} \mathbf{v}_{A}^{2}=q_{\text {in }}
$$

where $\mathbf{v}_{A}$ and $\mathbf{v}_{E}$ are the inlet and exit velocities of the air, as seen from the engine. Thus, from the viewpoint of an observer in the rest frame of the engine, the heat is used solely to increase the kinetic energy of the flow, and to heat it, while the engine does not produce work.

By introducing the propulsive power equation (7), the first law can be rewritten as

$$
h_{5}-h_{0}+\frac{1}{2}\left(\mathrm{v}_{E}-\mathrm{v}_{A}\right)^{2}=q_{i n}-\frac{\dot{W}_{P}}{\dot{m}} .
$$

This equation is appropriate for an observer resting in the environment, who observes resting air entering the engine, and a hot stream of velocity $\left(\mathbf{v}_{E}-\mathbf{V}_{A}\right)$ expelled into the environment. This observer sees the airplane moving with velocity $\mathbf{v}_{A}$ and recognises the propulsive power $\dot{W}_{P}$.

Since the outflow of the exhaust into the environment is not further utilised - it is simply dumped into the environment - the power loss to external irreversibilities is given by (note that $\mathrm{v}_{0}=0$ ) 


$$
T_{0} \dot{S}_{\mathrm{gen}}=\dot{m} \psi_{5}=\dot{m}\left(h_{5}-h_{0}-T_{0}\left(s_{5}-s_{0}\right)+\frac{1}{2}\left(\mathrm{~V}_{E}-\mathrm{V}_{A}\right)^{2}\right) .
$$

Obviously it is technically impossible to harvest any of the energy (or exergy) of the outflow from a moving plane, and thus one should aim at minimising the losses. This is tantamount to minimising the entropy generation $\dot{S}_{\text {gen }}$ of the equilibration between exhaust and environment.

We split the loss into its contributions from thermal and mechanical processes as

$$
\dot{W}_{\text {loss }}=T_{0} \dot{S}_{\text {gen }}=\dot{W}_{\text {thermal, loss }}+\dot{W}_{\text {mech,loss }}
$$

First we consider the mechanical loss: The fast outflow, which has the velocity $\left(\mathbf{v}_{E}-\mathbf{v}_{A}\right)$ with respect to the environment, mixes with the surrounding air. Friction and thermal processes will dissipate the kinetic energy and the amount of kinetic energy destroyed per unit time is $\dot{W}_{\text {mech,loss }}=\dot{m} / 2\left(\mathrm{~V}_{E}-\mathrm{V}_{A}\right)^{2}$. This loss should be minimised for the given propulsive power which is required to keep the plane airborne. This leads to the definition of the mechanical loss ratio as

$$
\chi_{\text {mech }}=\frac{\dot{W}_{\text {mech,loss }}}{\dot{W}_{P}}=\frac{\mathbf{v}_{E}-\mathbf{v}_{A}}{2 \mathbf{v}_{A}} .
$$

Similarly, we can define the thermal loss ratio

$$
\chi_{\text {thermal }}=\frac{\dot{W}_{\text {thermal loss }}}{\dot{W}_{P}}=\frac{h_{5}-h_{0}-T_{0}\left(s_{5}-s_{0}\right)}{\left(\mathrm{v}_{E}-\mathrm{v}_{A}\right) \mathrm{v}_{A}},
$$

and the overall loss ratio,

$$
\chi=\frac{T_{0} \dot{S}_{\text {gen }}}{\dot{W}_{P}}=\chi_{\text {thermal }}+\chi_{\text {mech }} .
$$

Efficient propulsion requires to minimise the loss ratio.

Before we discuss the loss ratios further, we recall the propulsive efficiency $\eta_{p}$ (Cumpsty, 2003), also known as Froude efficiency, which is defined as the ratio of propulsive power to the gain in kinetic energy of the flow (as observed from the engine)

$$
\begin{aligned}
& \dot{W}_{\text {kin }}=\frac{\dot{m}}{2}\left(\mathrm{v}_{E}^{2}-\mathrm{V}_{A}^{2}\right), \\
& \eta_{P}=\frac{\dot{W}_{P}}{\dot{W}_{\text {kin }}}=\frac{2 \mathrm{v}_{A}}{\mathrm{v}_{E}+\mathrm{v}_{A}}=\frac{1}{1+\chi_{\text {mech }}} .
\end{aligned}
$$

The Froude efficiency is the measure for air engine efficiency from the standpoint of fluid dynamics, it considers only mechanical energy. The mechanical loss ratio (equation (12)) is an equivalent measure. The total loss ratio (equation (14)), on the other hand, is an obvious measure from the standpoint of thermodynamics and second law analysis, it considers mechanical and thermal losses. 
From the mechanical loss measure it becomes evident that for efficient propulsion, one will aim for low loss ratio, or high propulsive efficiency. Propulsion is most effective when the outflow velocity $\mathbf{v}_{E}$ is not too far from the inflow velocity $\mathbf{v}_{A}$. Modern turbines with blade cooling allow large turbine inlet temperatures which translates into high exhaust velocity $\mathbf{v}_{E}$ and thus low efficiencies. In order to overcome this problem, modern aircraft engines have an additional turbine that drives a fan to move air through a bypass. This arrangement increases the mass flow through the engine and decreases the outflow velocity, and thus allows to produce enough propulsive power at higher efficiency. The fan is particularly important at take-off, where the power demand is high, but the airplane speed $\mathrm{v}_{A}$ is low.

\section{Detailed calculation for a reversible bypass engine}

In order to deepen the previous discussion we compute the loss ratio for a bypass engine. We consider a turbofan engine which works according to the series of processes detailed in Table 2 and Figure 2.

Table 2 Bypass engine processes

\begin{tabular}{lll}
\hline gas turbine, $\dot{m}_{G T}$ & & \\
$0-1$ & Adiabatic diffuser & $h_{1}=h_{0}+(1 / 2) \mathbf{v}_{A}^{2}$ \\
$1-2$ & Adiabatic compressor & $w_{C}=h_{1}-h_{2}$ \\
$2-3$ & Isobaric heating (combustion) & $q_{i n}=h_{3}-h_{2}$ \\
$3-4$ & Adiabatic turbine to drive compressor & $w_{T 1}=h_{3}-h_{4}=-w_{C}$ \\
$4-5$ & Adiabatic turbine to drive fan & $w_{T 2}=h_{4}-h_{5}=-\alpha w_{F}$ \\
$5-6$ & Adiabatic nozzle & $\mathrm{v}_{G T}=\sqrt{2\left(h_{5}-h_{6}\right)}$ \\
$f a n, \dot{m}_{F}=\alpha \dot{m}_{G T}$ & & \\
$0-1^{\prime}$ & Adiabatic diffuser & $h_{1^{\prime}}=h_{0}+(1 / 2) \mathbf{v}_{A}^{2}$ \\
$1^{\prime}-2^{\prime}$ & Adiabatic fan & $w_{F}=h_{1^{\prime}}-h_{2^{\prime}}$ \\
$2^{\prime}-3^{\prime}$ & Adiabatic nozzle & $\mathbf{v}_{F}=\sqrt{2\left(h_{2^{\prime}}-h_{3^{\prime}}\right)}$ \\
\hline
\end{tabular}

Here we have introduced the bypass ratio $\alpha$, defined as the ratio of the mass flows through the fan and the gas turbine in the core. In order to calculate the details of the processes, one has to specify some design parameters that the engineer can chose. For the following calculation these are: the temperature $T_{3}$, which is the maximum turbine inlet temperature; the pressure ratio $P=P_{2} / P_{0}$, which follows from the compressor characteristics; the bypass ratio $\alpha$, which is given by the ratio of the inlet cross sections; and, finally, the exit velocity of the fan, $\mathbf{v}_{F}$, which follows from the power developed by the turbine that drives the fan. 
Figure 2 Bypass jet engine

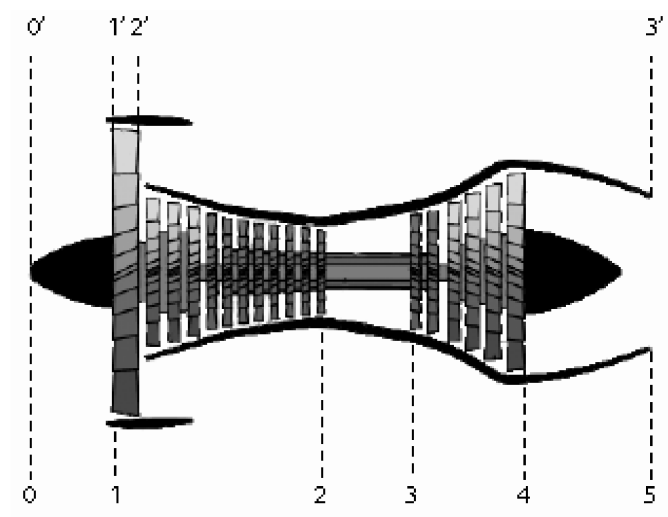

To simplify the computation, we assume the working fluid to be cold air as ideal gas (constant specific heats), so that

$$
h=c_{p}\left(T-T_{0}\right)+h_{0}, \quad s=c_{p} \ln \left[\frac{T}{T_{0}}\left(\frac{p}{p_{0}}\right)^{(1-k) / k}\right]+s_{0},
$$

where $c_{p}$ denotes the specific heat at constant pressure, and $k=c_{0} /\left(c_{0}-R\right)$ is the ratio of specific heats. Since we are concerned with external irreversibility it is convenient to assume that the engine is internally reversible; the relations for adiabatic reversible processes read $p v^{k}=$ const,$T p^{(I-k) / k}=$ const. We also make the simplification that fuel is not combusted directly with the air passing through the engine; therefore, the mass of the fuel which is added to the exhaust is neglected along with the change in the chemical exergy due to the change in composition between the exhaust and the environment; both factors are considered small (Turgut et al., 2007).

We consider the fan first: The flow $\left(0-3^{\prime}\right)$ is reversible and adiabatic throughout, and the inlet and exit pressures are equal, $p_{3^{\prime}}=p_{0}$. The adiabatic relation therefore gives $T_{3^{\prime}}=T_{0}$ which implies for the specific work to drive the fan

$$
w_{F}=-\frac{1}{2}\left(\mathbf{V}_{F}^{2}-\mathbf{V}_{A}^{2}\right)
$$

The exergy of the bypass exhaust is simply $\psi_{3^{\prime}}=\frac{1}{2}\left(\mathbf{v}_{F}-\mathbf{v}_{A}\right)^{2}$, since $T_{3^{\prime}}=T_{0}$ and $p_{3^{\prime}}=p_{0}$.

For the processes in the gas turbine, the thermodynamic laws and relations give the following relations for temperatures, pressures, and velocities,

$$
\begin{aligned}
& T_{1}=T_{0}+\frac{\mathbf{v}_{A}^{2}}{2 c_{p}}, \quad p_{1}=p_{0}\left(1+\frac{\mathbf{v}_{A}^{2}}{2 c_{p} T_{0}}\right)^{k /(k-1)} \\
& T_{2}=T_{0} P^{k-1 / k}, \quad p_{2}=P p_{0} \\
& q_{\text {in }}=c_{p}\left(T_{3}-T_{2}\right), \quad p_{3}=P p_{0}
\end{aligned}
$$




$$
\begin{aligned}
& T_{4}=T_{3}+T_{1}-T_{2}, \quad p_{4}=P p_{0}\left(\frac{T_{4}}{T_{3}}\right)^{k /(k-1)} \\
& T_{5}=T_{4}-\frac{\alpha}{2 c_{p}}\left(\mathbf{v}_{F}^{2}-\mathbf{v}_{A}^{2}\right), \quad p_{5}=P p_{0}\left(\frac{T_{5}}{T_{3}}\right)^{k /(k-1)} \\
& T_{6}=T_{3} P^{\frac{1-k}{k}}, \quad \mathbf{v}_{G T}=\sqrt{2 c_{p}\left(T_{5}-T_{6}\right)} .
\end{aligned}
$$

The above equations can be combined to give the exhaust velocity as

$$
\mathbf{v}_{G T}=\sqrt{2 c_{p}\left(T_{3}-T_{0} P^{(k-1) / k}\right)\left(1-P^{(k-1) / k}\right)-\alpha \mathbf{v}_{F}^{2}+(\alpha+1) \mathbf{v}_{A}^{2}} .
$$

The total thrust of the engine is

$$
F=\dot{m}_{G T}\left(\mathbf{v}_{G T}-\mathbf{v}_{A}\right)+\dot{m}_{F}\left(\mathbf{v}_{F}-\mathbf{v}_{A}\right),
$$

and its propulsive power is

$$
\dot{W}_{P}=\dot{m}_{G T}\left[\mathbf{v}_{G T}+\alpha \mathbf{v}_{F}-(1+\alpha) \mathbf{v}_{A}\right] \mathbf{v}_{A} .
$$

The external entropy generation loss due to equilibration of the exhaust with the environment assumes the form

$$
\begin{aligned}
T_{0} \dot{S}_{\mathrm{gen}} & =\dot{m}_{G T}\left(\psi_{6}+\alpha \psi_{3^{\prime}}\right) \\
& =\dot{m}_{G T}\left\{c_{p} T_{0}\left[\frac{T_{3}}{T_{0}} P^{\frac{1-k}{k}}-1-\ln \left(\frac{T_{3}}{T_{0}} P^{\frac{1-k}{k}}\right)\right]+\frac{1}{2}\left(\mathbf{v}_{G T}-\mathbf{v}_{A}\right)^{2}+\frac{\alpha}{2}\left(\mathbf{v}_{F}-\mathbf{v}_{A}\right)^{2}\right\}
\end{aligned}
$$

We can combine the above results into the total loss ratio

$$
\chi=\frac{c_{p} T_{0}\left[\frac{T_{3}}{T_{0}} P^{(1-k) / k}-1-\ln \left(\frac{T_{3}}{T_{0}} P^{(1-k) / k}\right)\right]+\frac{1}{2}\left(\mathbf{V}_{G T}-\mathbf{V}_{A}\right)^{2}+\frac{\alpha}{2}\left(\mathbf{V}_{F}-\mathbf{V}_{A}\right)^{2}}{\left[\mathbf{v}_{G T}+\alpha \mathbf{V}_{F}-(1+\alpha) \mathbf{V}_{A}\right] \mathbf{V}_{A}}
$$

The evaluation of the loss ratio (equation (21)) together with equation (17) clearly shows that the loss ratio decreases with increasing bypass ratio $\alpha$, when the other parameters are kept constant.

\section{Parametric studies}

A more detailed discussion requires the particular choices of the design parameters. We note that, for given $\alpha$, higher turbine inlet temperatures $T_{3}$ leads to a larger loss ratio. This, however, does not imply that $T_{3}$ should be low. The propulsive power, for the present case, is given by

$$
\dot{W}_{P}=\dot{m}_{G T}\left[\sqrt{2 c_{p}\left(T_{3}-T_{0} P^{\frac{k-1}{k}}\right)\left(1-P^{\frac{1-k}{k}}\right)-\alpha \mathbf{v}_{F}^{2}+(\alpha+1) \mathbf{v}_{A}^{2}}+\alpha \mathbf{v}_{F}-(1+\alpha) \mathbf{v}_{A}\right] \mathbf{v}_{A} .
$$

Obviously engines running at low $T_{3}$ require a larger mass flux to produce the same propulsive power. Since weight is a major issue for air engines, one tries to make the 
engines as light and small as possible, and larger $T_{3}$ allows for more compact engines. Therefore one will aim for high turbine inlet temperatures $T_{3}$; note that $T_{3}$ is constrained by the material properties of the turbine, and blade cooling.

We now have propulsive power (equation (22)) and total loss ratio (equation (21)) as functions of the design parameters $P, \alpha, \mathbf{v}_{F}$. In our analysis the airplane velocity $\mathbf{v}_{A}$ is not controllable, and for numerical examples and plots we consider values of $\mathbf{v}_{A}=290 \mathrm{~m} / \mathrm{s}$, $T_{0}=230 \mathrm{~K}, R_{\text {air }}=287 \mathrm{~J} / \mathrm{kgK}, c_{p}=1005 \mathrm{~J} / \mathrm{kgK}, k=1.4$ and $T_{3}=1500 \mathrm{~K}$.

To further reduce the parameter space, we first consider the conditions for maximum propulsive power, which read

$$
\frac{\partial \dot{W}_{P}}{\partial \mathbf{v}_{F}}=\frac{\partial \dot{W}_{P}}{\partial P}=\frac{\partial \dot{W}_{P}}{\partial \alpha}=0
$$

The first condition gives us the optimum fan exit velocity (maximal $\dot{W}_{P}$ ) as

$$
\mathbf{V}_{F \max }=\sqrt{\mathbf{V}_{A}^{2}+\frac{2}{1+\alpha} c_{p}\left(T_{3}-T_{0} P^{(k-1) / k}\right)\left(1-P^{(k-1) / k}\right)} .
$$

We substitute $\mathbf{v}_{F \max }$ into the power $\dot{W}_{P}$ (22), and then compute the optimal pressure ratio as

$$
P_{\max }=\left(\frac{T_{3}}{T_{0}}\right)^{k / 2(k-1)}
$$

The resulting pressure ratio $P_{\max }$ is independent of $\alpha$, and, indeed, equal to the optimal pressure ratio for maximum work in a simple gas turbine power cycle (Cengel and Boles, 2002; Moran and Shapiro, 1999). The above values for $T_{0}$ and $T_{3}$ yield the optimal pressure ratio as $P_{\max }=26.6$.

With the results for $P_{\max }$ and $\mathbf{v}_{F \max }$ substituted, the expressions for loss ratio $\chi(21)$ and propulsive power equation (22) become functions of $\alpha$ alone.

Moreover, with $P_{\max }$ the optimum fan exit velocity equation (24) and the gas turbine exit velocity equation (17) are equal, $\mathbf{v}_{F \max }=\mathbf{v}_{G T \max }$; as $\alpha$ becomes large, they both approach $\mathbf{v}_{A}$. This implies that more power is achieved when the turbine is used to raise the fan exit velocity rather than expel the gas turbine exhaust at high speed; ideally both velocities are equal. The velocity is plotted in Figure 3 as function of the bypass ratio $\alpha$. As expected, the exhaust velocity decreases with increasing bypass ratio.

As discussed above, lower exhaust velocities should reduce the external losses, and therefore we proceed with investigating the impact of the bypass ratio on loss ratio and propulsive power. Figure 4 shows the loss ratio $\chi(21)$ as function of the bypass ratio $\alpha$ (again with $\mathbf{v}_{F}=\mathbf{v}_{F \max }$ and $P=P_{\max }$ ).

The Figure 4 shows that increasing the bypass ratio minimises the loss ratio; hence this minimises the waste of energy. The impact of $\alpha$ is most dramatic in the interval $[0,5]$, where the curve has its steepest descent. Indeed, commercial high-bypass engines tend to exceed that range. e.g., the Pratt and Whitney PW4000 series, commonly used by Boeing and Airbus, has bypass ratios between 4.8-6.4. 
Figure 3 Exit velocity of fan and gas turbine under optimal conditions, $\mathbf{v}_{F \max }$, as a function of bypass ratio $\alpha$

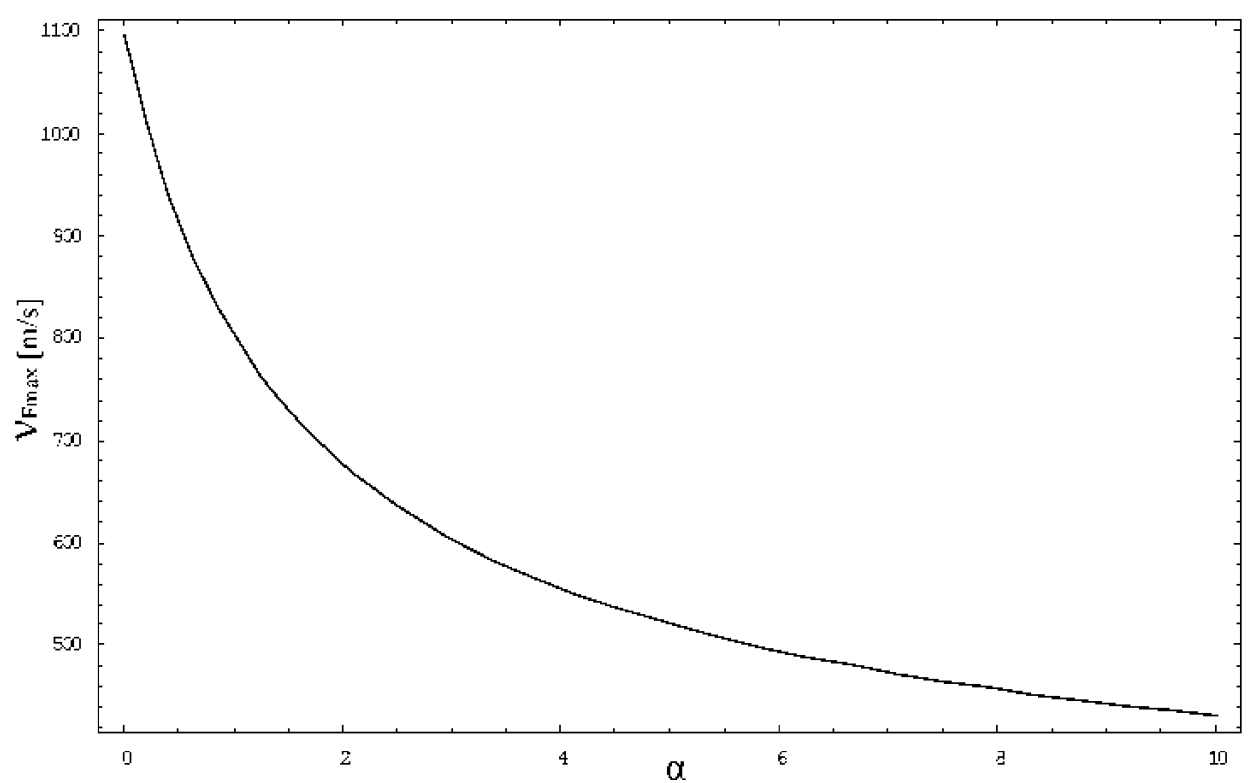

Figure 4 Loss ratio $\chi$ as a function of bypass ratio with $P_{\max }$ and $\mathbf{v}_{F \max }$ (maximum propulsive power)

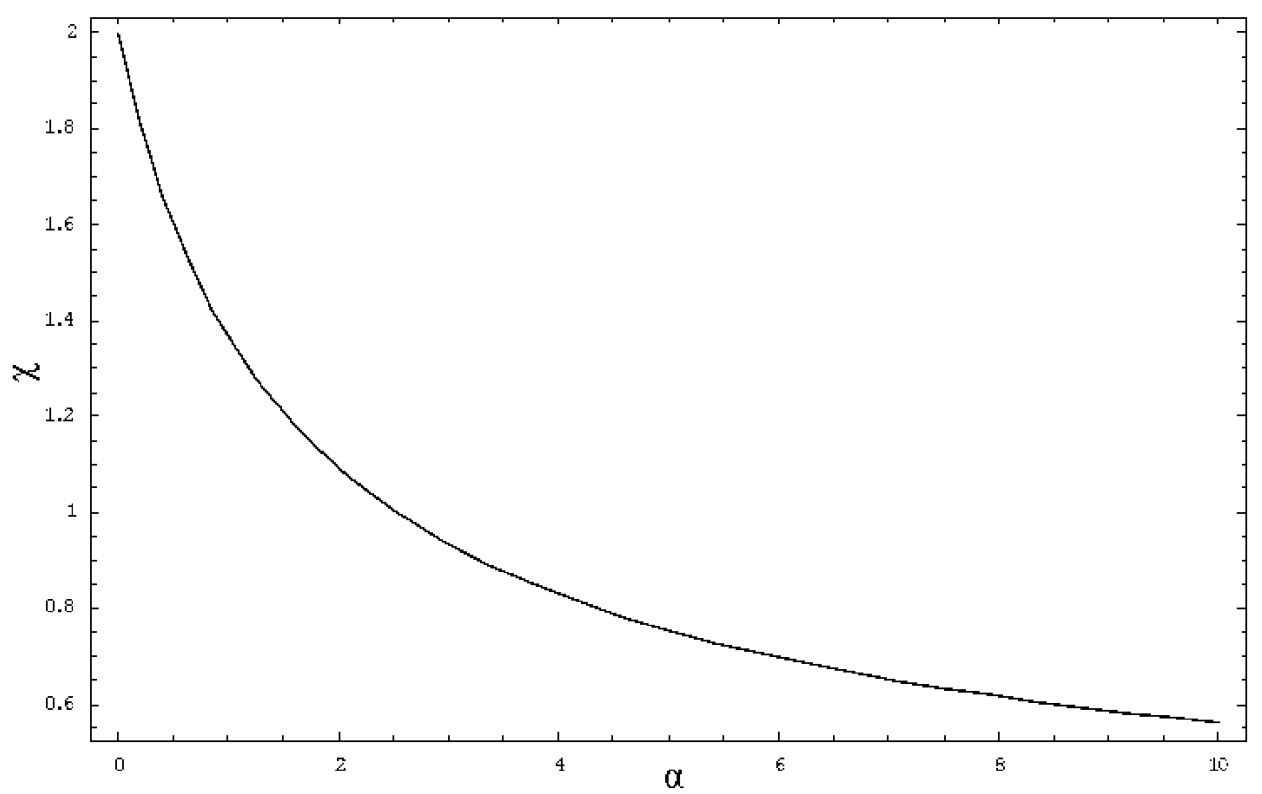


High efficiency, or small loss ratio, is highly desirable, but of no value without sufficient propulsive power. Figure 5 shows the specific propulsive power per unit mass flowing through the gas turbine, $W_{P}=\dot{W}_{P} / \dot{m}_{G T}$, as function of $\alpha$ (with $\mathbf{v}_{F}=\mathbf{v}_{F \max }$ and $P=P_{\max }$ ).

Figure 5 Specific propulsive work per unit mass flowing through the gas turbine, $W_{P}=\dot{W}_{P} / \dot{m}_{G T}$

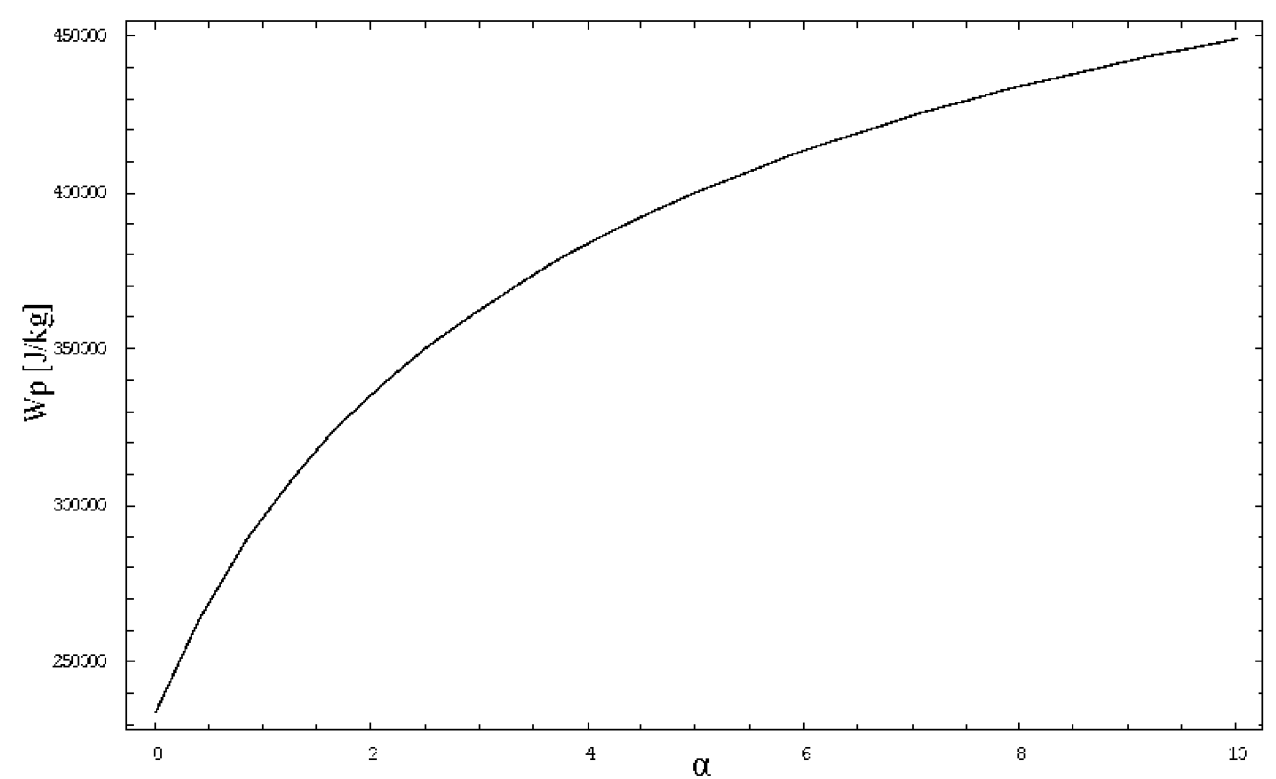

Increase of the bypass ratio yields more propulsive power for a given mass flow. Again, the increase is more pronounced in the interval $[0,5]$. We conclude that high bypass ratios reduce irreversible losses outside the engine and increase the specific power output.

Next we consider the engine size: If we set the diameter $D$ of the total engine to be a constant, the mass flow through the gas turbine is given by

$$
\dot{m}_{G T}=\rho_{\text {air }} A_{G T} \mathbf{v}_{A}=\frac{p_{0}}{R_{\text {air }} T_{0}} \frac{\pi D^{2}}{4(1+\alpha)} \mathbf{v}_{A}
$$

Figure 6 shows the total propulsive power $\dot{W}_{P}$ for a engine with 100 inches diameter.

An increase of the bypass ratio $\alpha$ leads not only to a reduced mass flow through the turbine, $\dot{m}_{G T}$, but also to a decrease in propulsive power of the engine. For applications which require a compact size, and a maximum of power without attention to efficiency, a lower bypass ratio is desirable; this, indeed, is the case in military applications. For a commercial or transport air craft, however, where fuel efficiency is critical, we see a trend towards larger engines with higher bypass ratios. The combination of large turbine inlet temperature $T_{3}$ and large bypass ratio provides the optimum compromise between efficiency and weight. 
Figure 6 Propulsive power $\dot{W}_{P}$ as a function of bypass ratio for fixed diameter $D$

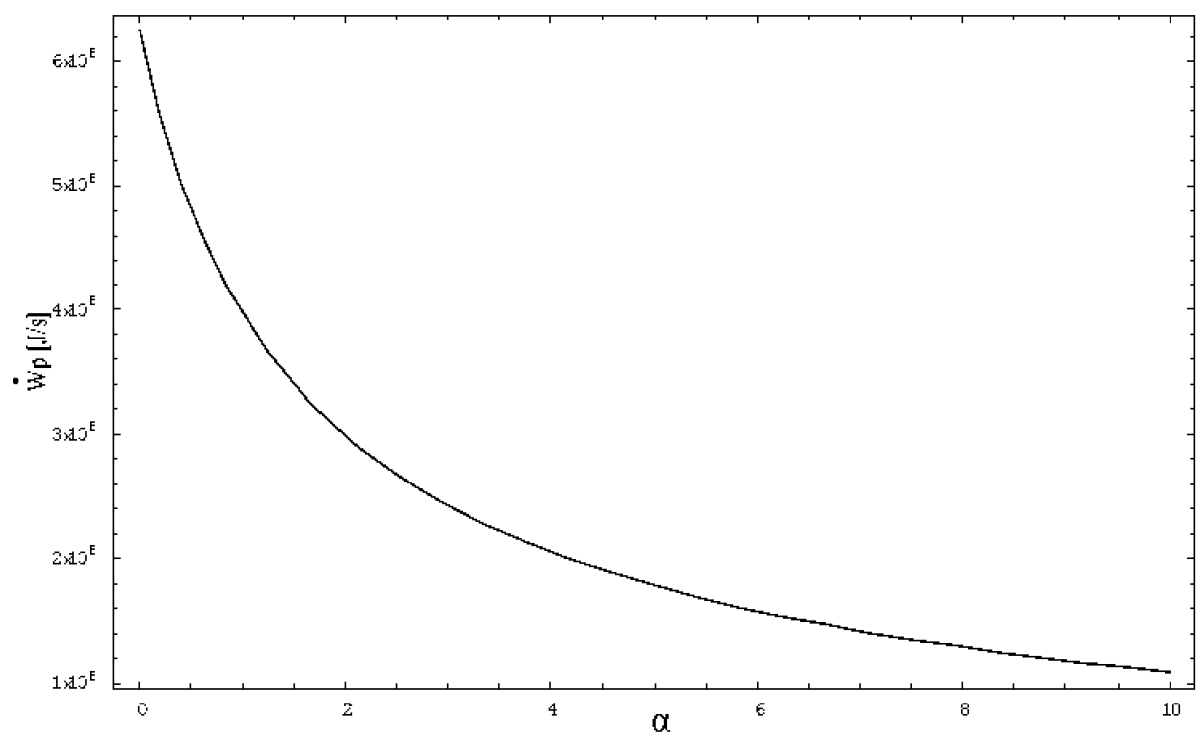

\section{Conclusions}

We have presented an analysis of the external losses in aircraft gas turbines due to irreversibilities that provides a thermodynamic argument in favour of bypass engines. We also outlined the basic calculation of the processes in a bypass air engine. The calculations show that a bypass ratio of more than five should be used.

\section{Acknowledgements}

This research was supported by the Natural Sciences and Engineering Research Council (NSERC). The paper was written during HS's stay as Gastprofessor at ETH Zürich, and he would like to thank Prof. Hans Christian Öttinger and his group at the Institute for Polymer Physics for their kind hospitality.

\section{References}

Cengel, Y.A. and Boles, M.A. (2002) Thermodynamics-An Engineering Approach, McGraw-Hill, Boston.

Cumpsty, N. (2003) Jet Propulsion: A Simple Guide to the Aerodynamic and Thermodynamic Design and Performance of Jet Engines, 2nd ed., Cambridge University Press, Cambridge.

Moran, M.J. and Shapiro, H.N. (1999) Fundamentals of Engineering Thermodynamics, Wiley, New York.

Ordonez, J.C. and Bejan, A. (2003) 'Minimum power requirement for environmental control of aircraft', Energy, Vol. 28, pp.1183-1202. 
Paulus Jr., D.M. and Gaggioli, R.A. (2003) 'The exergy of lif and aircraft exergy flow diagrams', Int.J. Thermodynamics, Vol. 6, No. 4, pp.149-156.

Sonntag, R.E., Borgnakke, C. and van Wylen, G.J. (2003) Fundamentals of Thermodynamics, Wiley, New York.

Turgut, E.T., Karakoc, T.H. and Hepbasli, A. (2007) 'Exergetic analysis of an aircraft turbofan engine', Int. J. Energy Res (in press), Published Online: 5 Mar 2007, DOI: 10.1002/er.1310.

\section{Nomenclature}

\begin{tabular}{|c|c|}
\hline$c_{p}$ & Specific heat at constant pressure \\
\hline$F$ & Thrust \\
\hline$h$ & Specific enthalpy \\
\hline$k$ & Ratio of specific heats \\
\hline$\dot{m}$ & Mass flow \\
\hline$p$ & Pressure \\
\hline$P$ & Pressure ratio \\
\hline$q$ & Specific heat exchange \\
\hline$\dot{Q}$ & Heat exchange \\
\hline$s$ & Specific entropy \\
\hline$\dot{S}_{\mathrm{gen}}$ & Entropy generation rate \\
\hline$T$ & Temperature \\
\hline $\mathbf{V}$ & Velocity \\
\hline$W$ & Specific work \\
\hline$\dot{W}$ & Power \\
\hline \multicolumn{2}{|c|}{ Greek letters } \\
\hline$\alpha$ & Bypass ratio \\
\hline$\chi$ & Loss ratio \\
\hline$\psi$ & Specific flow exergy \\
\hline$\eta_{p}$ & Froude efficiency \\
\hline \multicolumn{2}{|c|}{ Subscripts } \\
\hline$A$ & Aircraft \\
\hline$C$ & Compressor \\
\hline$e, E$ & Exit \\
\hline$F$ & Fan \\
\hline$G T$ & Gas turbine \\
\hline$i$ & Inlet \\
\hline$T$ & Turbine \\
\hline 0 & Environment \\
\hline
\end{tabular}

